Vietnam Journal of Mechanics, VAST, Vol.34, No. 4 (2012), pp. 281-288

\title{
DEVELOPMENT OF HYDROGEL-BASED MEMS PIEZORESISTIVE SENSORS FOR DETECTION OF SOLUTION PH AND GLUCOSE CONCENTRATION
}

\author{
Trinh Quang Thong ${ }^{1}$, Margarita Guenther ${ }^{2}$, Gerald Gerlach ${ }^{2}$ \\ ${ }^{1}$ Hanoi University of Science and Technology, Vietnam \\ ${ }^{2}$ Dresden Technical University, Germany
}

\begin{abstract}
Hydrogels are water-swollen polymeric materials that maintain a distinct three-dimensional structure. Manipulation of hydrogel structure has produced stimulisensitive hydrogels, which change their swelling degree or undergo phase transition in response to minimal changes in environmental conditions. This paper presents the development of hydrogel-based sensors for measurement of solution $\mathrm{pH}$ and glucose concentration using commercial MEMS piezoresistive pressure transducers. $\mathrm{pH}$-sensitive PVA/PAA and glucose-sensitive AAm/3-APB/BIS hydrogels are used in the experiments. Two types of sensor packaging, one is used as solution container and the other used to dip into solution have been performed. The measurements for different solutions having $\mathrm{pH}$ values between 1 to 11 and glucose concentrations between $2.5 \mathrm{mM}$ and $15 \mathrm{mM}$ have been carried out. The output characteristics of sensors have been demonstrated showing the long-term reproduction and relatively good sensitivity, namely approximately $20 \mathrm{mV} / \mathrm{pH}$ for $\mathrm{pH}$ sensor and $7 \mathrm{mV} / 1 \mathrm{mM}$ of glucose concentration for glucose sensor.
\end{abstract}

Keyword: Chemical sensors, MEMS piezoresistive senors, polymer hydrogels.

\section{INTRODUCTION}

Over the years, the field of chemical and bio-sensing has evolved from simple and bulk systems to more reliable, robust and low cost microscale systems that can perform complex operations minimizing human efforts and attaining highly accurate results. These Microelectromechanical Systems (MEMS) based sensors have not only been used in industrial and automobile sectors but also have a rapid growth in the field of medical diagnostics [1].

$\mathrm{pH}$ is one of the most important chemical measurands in laboratories and industry and plays an important role in our life. Up to date several types of sensors have been developed for measuring $\mathrm{pH}$ such as electrochemical (EC) sensors [2], optical sensors [34], and ISFETs [5-6]. Both EC and ISFETs-based sensors need the referent electrode. For EC sensors it is difficult in remote sensing and miniaturization. The optical sensors have narrow dynamic range and may be influenced by light ambient. The development of glucose sensors is also an area of intense research with application in diabetes, obesity and metabolic monitoring in general. As of now, the standard method for glucose measurements in diabetes patients is the blood finger-prick test using the sensor strip read by a meter 
displaying the glucose concentration within seconds [7-9]. Nevertheless, the glucose levels are measured in the extract of the interstitial fluid which has much lower concentrations of glucose than the body. In order to properly monitor sugar levels within the body, this method must be performed multiple times daily which leads to a lot of complications. Other sensors have a glucose oxidase based electrode also developed [10]. However, these sensors have their particular drawbacks including short life span and need calibration at regular short intervals [11-12]. Both types of above $\mathrm{pH}$ and glucose sensors are limited long-term stability, short life-time and require the complicated readout circuits.

The sensors presented here exploit the swelling property of polymer hydrogels and the corresponding deformation of a flexible thin diaphragm within the pressure transducer to measure the change in $\mathrm{pH}$ level and glucose concentration. The devices are expected to have the advantages such as simplicity for measurement, ease for fabrication and purpose of miniaturization as well as possibility eliminating the drawbacks of the studied sensors in the past.

\section{EXPERIMENTAL METHOD}

\subsection{MEMS piezoresistive pressure chip}

For the design and fabrication of the chemical sensor, commercially MEMS piezoresistive pressure chips were used. Usually, the silicon sensor chips possess four integrated resistors in a Wheatstone bridge within a thin plate (also called diaphragm). These are mechano-electrical transducers that transform the mechanical stress into electrical output signal. Generally, the resistors are located close to the diaphragm edges where the stress due to the external load is highest. For a square diaphragm, two resistors are positioned parallel and two others oriented perpendicular to the diaphragm edge as shown in Fig. 1. According to this configuration, the resistances of diagonally opposed legs vary equally

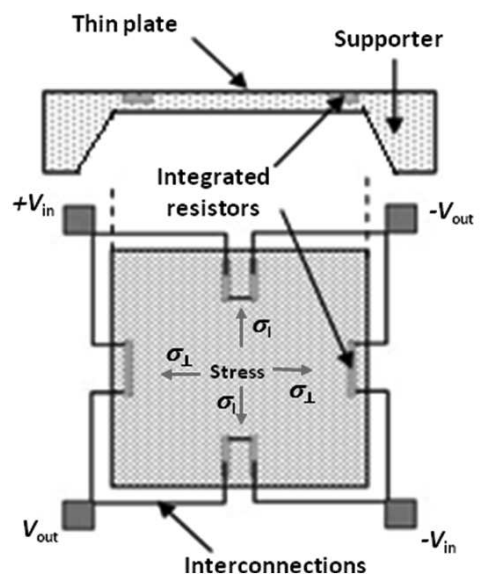

Fig. 1. Structure of MEMS piezoresistive pressure chip 
and in the same direction, as the resistance of diagonally opposed legs increases, the resistance of the other set decreases, when the thin plate is deformed. Then, according to the piezoresistive effect, the output voltage difference will be proportional to the longitudinal $\left(\sigma_{l}\right)$ and transverse $\left(\sigma_{t}\right)$ stresses resulting from the deformation of the thin plate [13].

\subsection{Hydrogel materials for detection of solution $\mathrm{pH}$ and glucose concentration}

Hydrogels (or gels) are the network of polymer molecules holds the liquid in place and so keeps its rigidity. Their features are wet and soft and look like solid material, but are capable of undergoing large deformations. This is in contrast with most industrial materials, such as metals, ceramics, and plastics, which are dry and hard. In other words, a hydrogel is a polymeric material that absorb a significant fraction of water within its structure, but that will not dissolve in water [14]. Structures of this type can be obtained by chemical or physical processes. Gels can easily be deformed by external physical and chemical stimuli and is able to generate a force or execute work to the exterior [15]. If such responses could be translated from the microscopic level into a macroscopic scale, a conversion of chemical free energy into mechanical work might be realized.

In this case, a blend of polyvinyl alcohol and polyacrylic acid (PVA/PAA) with the per dent ratio by weight as $80: 20 \%$ is used as pH-sensitve element. It is an acidic hydrogels which will swollen at high $\mathrm{pH}$, and unswollen at low $\mathrm{pH}$. The prepared samples of PVA/PAA are soaked in deionized water to get an equilibrium state of swelling and then preserved in buffer $\mathrm{pH} 1$ solution to be in the least swollen state [16]. In this case, buffer $\mathrm{pH}$ solution is a mixture of pure acetic acid $\left(\mathrm{HC}_{2} \mathrm{H}_{3} \mathrm{O}_{2}\right)$ and sodium acetate $\left(\mathrm{C}_{2} \mathrm{H}_{3} \mathrm{O}_{2-}\right)$ with $\mathrm{pH}$ values modified by adding either hydrochloric acid or sodium hydroxide.

For measurement of glucose concentration, a cross-linked glucose-sensitive copolymer of acrylamide and 3-acrylamidophenylboronic acid (AAm/3-APB) combined with $N, N$-methylenebisacrylamide (BIS, Sigma-Aldrich) at a nominal mole ratio of 80/20/0.25 is used in the present work [17-18]. The glucose hydrogel slabs were conditioned in 0.15 M Phosphate Buffered Saline (PBS) for 2 days prior to inserting in the cavities. PBS solution here is a water-based salt solution containing either sodium or potassium chloride, and sodium or potassium phosphate, which is commonly used in biological research. The buffer's phosphate groups help to maintain a constant $\mathrm{pH}$ at the value of 7.4. $\mathrm{D}(+)$-glucose (Roth) was dissolved in PBS solutions of ionic strength $\mathrm{I}=0.15 \mathrm{M}$. The osmotic pressure and ion concentrations of the solution exploited in this work would match those of the human body for the aim of medical applications afterwards.

\subsection{Sensor structure and measurement principle}

Piezoresistive bio-chemical sensors operate by monitoring the analyte-induced volume expansion of a thin polymer layer used as chemo-mechanical transducer. For the design of the chemical sensor, commercially available pressure sensor chips with the dimension of $5.00 \times 5.00 \times 0.400 \mathrm{~mm}^{3}$ were used. The measurement of concentrations of chemical species is performed in aqueous solutions. A slab of polymeric hydrogel with the thickness of $40 \mu \mathrm{m}$ is confined into the cavity at the backside of the silicon chip closed with a silicon grid allowing the solution and analyte molecules entering into the gel (Fig. 2 ). The grid is also made of silicon. Gel's induced swelling or shrinking processes lead to the plate's deflection causing a stress inside the plate and therefore a change of the 
resistivity of the piezoresistors affecting proportionally the output voltage of the sensor as demonstrated in Fig. 3. An increasing value of output voltage corresponds to a hydrogel swelling, whereas a decreasing one corresponds to a deswelling.

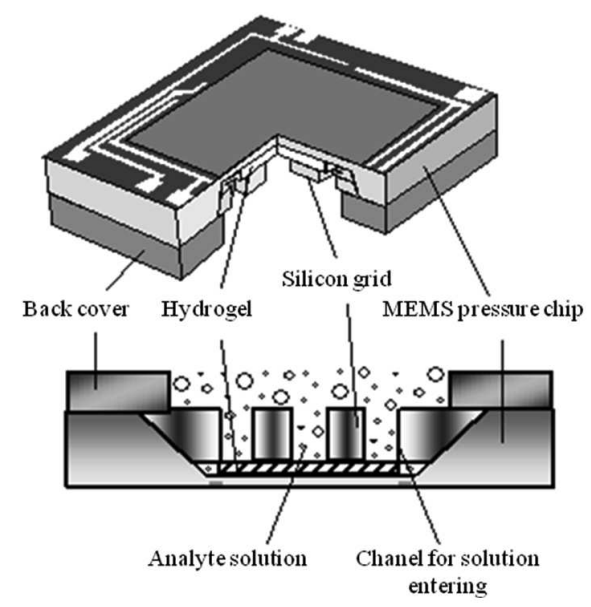

Fig. 2. Structure of hydrogel-based sensors for detection of change in solution $\mathrm{pH}$ and glucose concentration

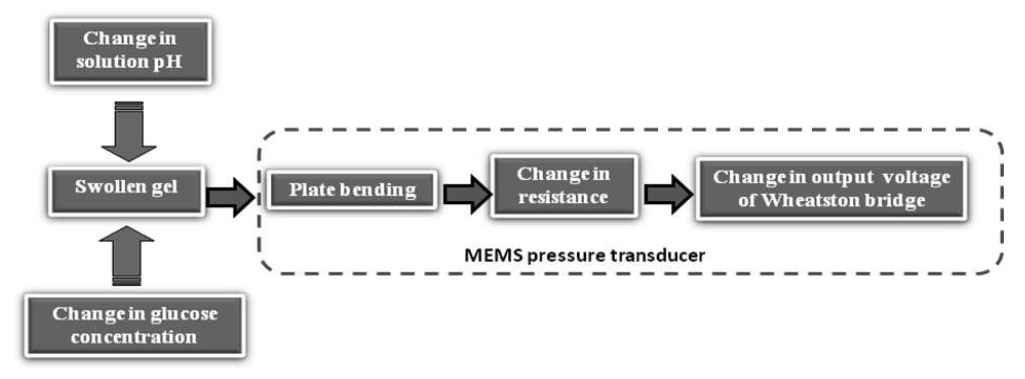

Fig. 3. Operational principle of sensors

Two types of sensor packaging have been performed. One is assembled as a solution container (Fig. 4a).that the solution is pumped into and drawn out of the pressure chip's cavity containing the gel inside. This type of sensor was used mostly in the reported studies [17-19]. The other is as the batch configuration (Fig. 4b) which can be dipped into a beaker with measured or calibrated solution. In this case, the sensor chip is defended by a cover to prevent the contact from the solution. It is considered to be an implemented design compared to the mentioned former one.

For the measurement, the sensor is supplied by an excitation voltage by a voltage of $5.00 \pm 0.05 \mathrm{~V}$. The output signal of sensor is connected to a data acquisition system consisting of a scanner (PREMA 2024) used to collect the output voltage signals of simultaneously several sensors and a digital multimeter (Keithley 2000) used to display the 


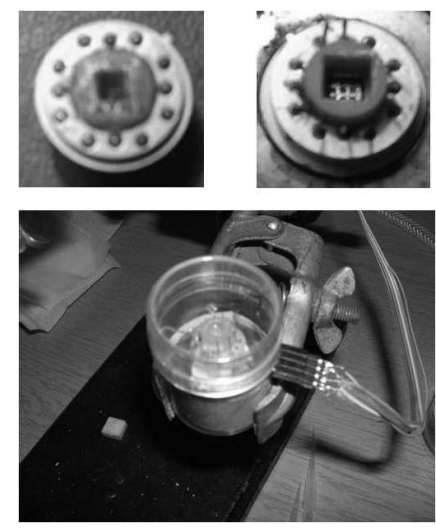

(a)

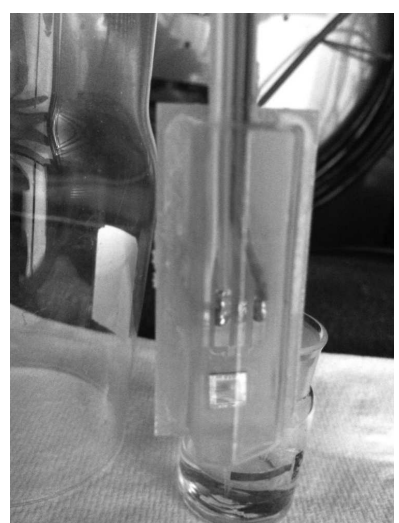

(b)

Fig. 4. Sensor packaging for test, $(a)$ solution container and $(b)$ bath configuration

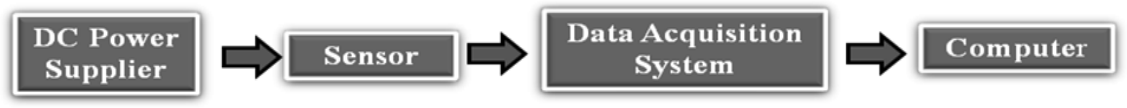

Fig. 5. Schematic showing the process flow diagram of the measurement setup

output voltage. The multimeter is then connected to a computer where the measurement date is acquired by means of a Labview program. The flow diagram of measurement set up is presented in Fig. 5 .

\section{RESULTS AND DISCUSSION}

\subsection{Detection of change in solution $\mathrm{pH}$}

The gel hydration state in $\mathrm{pH} 1$ solution was chosen as reference state. The measurement cycles have been performed using step-wise $\mathrm{pH}$ excitation. Fig. 6 is one of the measurement results obtained by the investigations. Here, the measurement was performed using the solutions in the dynamic $\mathrm{pH}$ range of 5 to 11 because the volume transition of PVA/PAA hydrogel occurs when the $\mathrm{pH}$ value rises above the dissociation constant $p K_{a}$ of PAA $\left(p K_{a}=4.7\right)$ that is between $\mathrm{pH} 4$ and $\mathrm{pH} 5$ [16]. It means that the ionization process of polycarboxylic groups within the polymer network happens and creates the electrostatic repulsion force leading to a significant increase of swelling. For each measurement cycle, the measurement results showed a slight hysteresis. This behavior is due to the screening effect of polymer hydrogels that was elucidated in previous works [20, 21]. The output voltage response for this type of sensor is in the range of 75 to $130 \mathrm{mV}$ corresponding to a $\mathrm{pH}$ range of 5 to 11. Based on long-term measurement, the sensor sensitivity is approximately estimated by a $20 \mathrm{mV} / \mathrm{pH}$ within this dynamic range of $\mathrm{pH}$ value. 


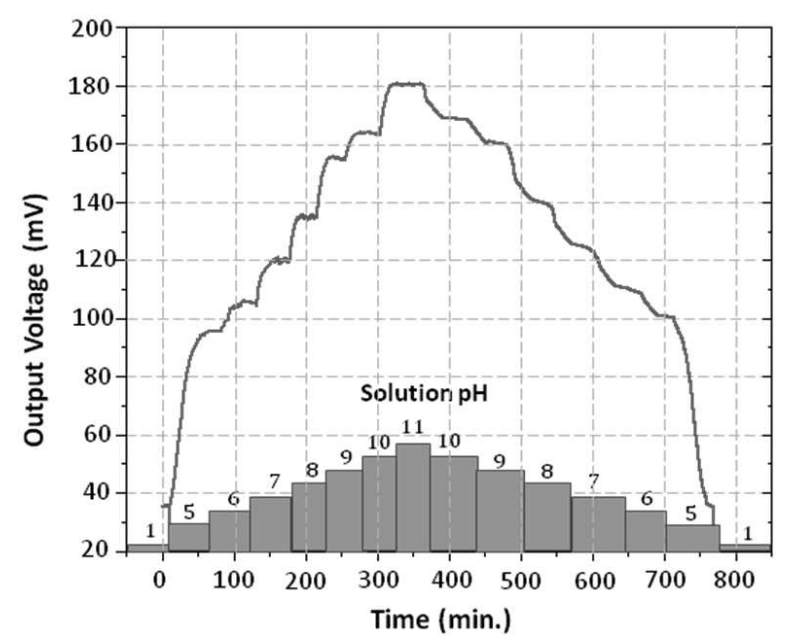

Fig. 6. Output characteristics of of $\mathrm{pH}$ sensor in loop measurement

\subsection{Detection of change in glucose concentration}

The sensor output voltage at steady state of the swollen gel in a glucose-free PBS buffer was determined and then used as reference value. The testing was performed with the sensor being immersed in glucose free $0.15 \mathrm{M}$ PBS at physiological pH (7.4) till the output signal attained stability. The concentrations of PBS are between $15 \mathrm{mM}$ and 2.5 $\mathrm{mM}$ in steps of decreasing concentration. The process was repeated in a reverse order to mirror the response and to complete a full cycle. A typical measurement result is given in Fig. 7. The initial rate of the volume increase depends on the step value of the gradual glucose concentration change. The change of the glucose concentration in PBS solution

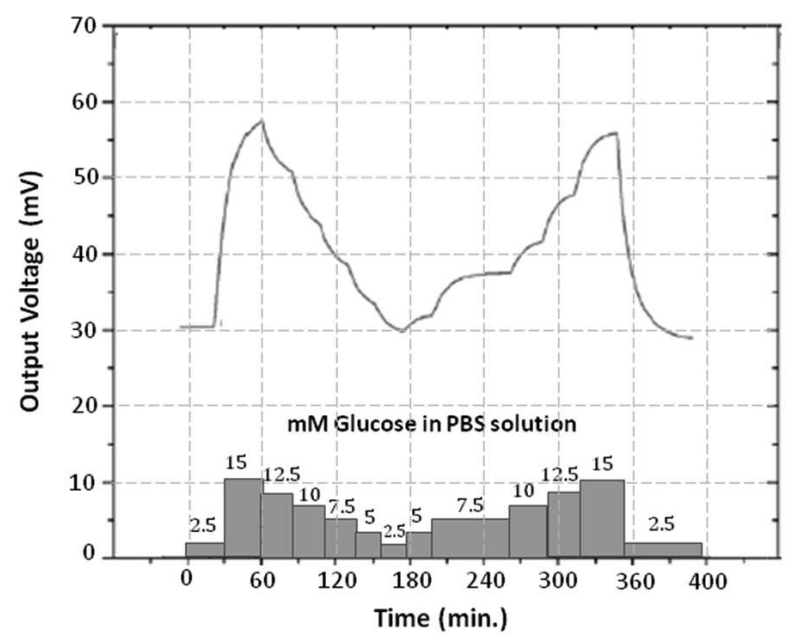

Fig. 7. Output characteristics of glucose sensor in loop measurement 
results in the swelling process of the glucose-sensitive AAm/3-AAmPBA hydrogel showing the volume increase with increasing glucose concentration in PBS solution. The sensitivity of glucose sensor is determined between $4 \mathrm{mV}$ to $7 \mathrm{mV}$ per $\mathrm{mM}$ glucose in PBS solution.

Obviously, for both the swelling and deswelling process, the glucose-sensitive gels quickly get stable state result in smaller output signal consequently the greater sensitivity as well as better time constant compared to those of $\mathrm{pH}$ sensors.

\section{CONCLUSIONS}

The MEMS based devices market is growing rapidly and widely exploited for reliable, low cost applications used in the field of bio-chemical sensors. In this work the piezoresistive micropressure sensors were used as the sensing platform with analyte specific stimuli response hydrogels as the sensory material for chemical sensing. The tests have been carried out in vitro for $\mathrm{pH}$ and glucose sensors based on $\mathrm{pH}$-sensitive PVA/PAA and glucose-sensitive copolymer of acrylamide and 3-acrylamidophenylboronic acid hydrogels. The response of the both types of sensor was studied at step changes in solutions. Not only the measuring principle was adapted to the investigated objects in aqueous solutions but also the implemented design of sensors has been used as an instrument for on-line monitoring of the gel behaviour kinetics. The long-term measurements in this work have shown that the life time of piezoresistive biochemical sensors under harsh physiological conditions can be prolonged up to long duration. Sensor sensitivity of both types of device also has been determined using the obtained measurement results that is about tens milivolts per $\mathrm{pH}$ value for $\mathrm{pH}$ sensors and several milivolts per glucose $\mathrm{mM}$ for glucose ones. Sensor response time was estimated that of glucose sensors is faster compared to $\mathrm{pH}$ ones. The experimental results are promising to develop the bio-sensors for applications of metabolic monitoring and medical diagnostics in the next research step.

\section{REFERENCES}

[1] Mohamed Gad-el-Hak, MEMS Applications of The MEMS Handbook serries, CRC Press, Taylor and Francis Group, (2006).

[2] Bard A. J., Faulkner L. R., Electrochemical Methods, Fundamentals and Applications, (1980).

[3] Wolfbeis O. S., Fiber Optic Chemical Sensors and Biosensors, CRC Press, Boca Raton,Vol. I, (1991).

[4] Elster J. L., Long period grating-based $\mathrm{pH}$ sensors for corrosion monitoring, Master thesis, Virginia Polytechnic Institute, Virginia State University, Blacksburg, Virginia, (1999).

[5] Bartic C., Palan B., Campitelli A., Borghs G., Monitoring pH with organic-based field-effect transistors, Sensors and Actuators B, 83, (2002), 115-122.

[6] Bergveld P., ISFETs: Theory and Practical, IEEE conference Toronto Canada, October 2003.

[7] Williams D. L., Doig A. R., Korosi A., Electrochemical-enzymatic analysis of blood glucose and lactate, Analytical Chemistry, 42, (1970), pp. 118-121.

[8] Newman J. D., Turner A. P., Home blood glucose biosensors: a commercial perspective, Biosens Bioelectron, 20, Jun 15 (2005), pp. 2435-53, .

[9] Koschwanez H. E.,Reichert W. M., In vitro, in vivo and post explantation testing of glucosedetecting biosensors: current methods and recommendations, Biomaterials, 28, (2007), pp. $3687-703$. 
[10] Deshpande D., Development of a nanowire based titanium needle probe sensor for glucose monitoring (Phd Dissertation), University of Arkansas, (2009).

[11] Oliver N. S., Toumazou C. , A. E. G. Cass, and D. G. Johnston, Glucose sensors: a review of current and emerging technology, Diabetic Medicine, 26, (2009), pp. 197-210.

[12] Wilson G. S. Z., Y, Introduction to the glucose sensing problem, in In Vivo Glucose Sensing, Hoboken, NJ: John Wiley, (2010).

[13] Gerlach G., Werthschuetzky R., 50 years of piezoresistive sensors - history and state of the art of piezoresistive sensors, Technisches Messen, 72, (2005), 53-76.

[14] Osada Y., Gong Y., Tanaka, Polymer gels, Journal of Macromolecular Science C, 44, (2004), p.87.

[15] Kopecek J.,Yang J., Hydrogels as smart biomaterials, Polymer International, 56, (2007), pp. 1078.

[16] Trinh Quang Thong, Hydrogel-based Piezoresistive pH Sensors, ISBN 3-938863-71-4, TUD Press - Verlag der Wissenschaften GmbH, Germany, (2006).

[17] Guenther M., Gerlach G., et.al, Biochemical microsensors on the basis of metabolically sensitive hydrogels, Electroactive Polymer Actuators and Devices (EAPAD) 2011, Federico Carpi, Proc. of SPIE Vol. 7976, 79762D 2011 SPIE, doi: 10.1117/12.880460.

[18] Guenther M., Gerlach G., et.al, Hydrogel-Based Biochemical Sensors, In proceedings of SENSOR+TEST Conferences 2011, p. 211.

[19] Gerlach G., Arndt K.-F.(eds.), Hydrogel Sensors and Actuators, Springer Series on Chemical Sensors and Biosensors, 6, (2009), DOI: 10.1007/978-3-540-75645-3_5, Springer-Verlag Berlin Heidelberg.

[20] Arndt K.-F., Richter A., Ludwig S., Zimmermann J., Kressler J., Kuckling D., Adler H.J., Poly(vinyl alcohol)/poly(acrylic acid) hydrogels: FT-IR spectroscopic characterization of crosslinking reaction and work at transition point, Acta Polymerica, 50, (1999), 383-390.

[21] Marra S. P., Ramesh K. T., Douglas A. S., The actuation of a biomimetic poly(vinyl alcohol)poly(acrylic acid) gel, Philosophical Transactions of the Royal Society of London, Series A, The Royal Society, 360, (2002), 175-198. 